\title{
A Note on a Group Preference Axiomatization with Cardinal Utility
}

\author{
Luis C. Dias \\ Faculty of Economics, University of Coimbra, 3004-512 Coimbra, Portugal; and \\ INESC Coimbra, 3000-033 Coimbra, Portugal, lmcdias@fe.uc.pt \\ Paula Sarabando \\ Polytechnic Institute of Viseu, 3504-510 Viseu, Portugal; and INESC Coimbra, 3000-033 Coimbra, Portugal, \\ psarabando@mat.estv.ipv.pt
}

\begin{abstract}
Kenneth Arrow's work on social welfare proposed a set of conditions that a function to aggregate ordinal K preferences of the members of a group should satisfy, proving that it was not possible to satisfy all these assumptions simultaneously. Later, Ralph Keeney adapted these conditions and proposed a cardinal utility axiomatization for the problem of aggregating the utility functions. This note discusses in particular the condition of nondictatorship. It proposes stronger formulations for this condition to limit the maximum influence that an individual can have, and it presents the corresponding characterization of compliant group cardinal utility functions. An extension to address coalitions of individuals acting strategically is also discussed.
\end{abstract}

Key words: group utility functions; multiattribute utility theory; additive model

History: Received on February 6, 2012. Accepted on April 9, 2012, after 1 revision. Published online in Articles in Advance July 18, 2012.

\section{Introduction}

Decision analysis is often called for to support decisions made by a group (e.g., a society, a committee, or a team). This can be accomplished by aggregating the utilities of the individuals by a group utility function, or by using other means of aggregating the results of an individual decision analysis, or by fostering discussion of these individual analyses, as discussed by Bose et al. (1997), not to mention the cases where decision analysis is not used at all (Schein 1999, pp. 158-164). A review by Keefer et al. (2004) mentions 12 decision analysis articles in the period 1990-2001 addressing group decision or the combination of expert opinions. For a recent review of the field of group decision in general, we refer the reader to Kilgour and Eden (2010).

This work focuses on the possibility of building a group utility function from individual utility functions, rather than situations where a group gets together to conjointly build a model reaching a consensus (for examples of the latter, see Phillips and Bana e Costa 2007, Merrick et al. 2005). There are several proposals of axioms for characterizing a group utility function. As examples we can cite Harsanyi (1955), Keeney and Kirkwood (1975), and Baucells and Sarin (2003) for the case of von Neumann and Morgenstern (1947) utilities, or Dyer and Sarin (1979) and Harvey (1999) for the case of utilities based on strength of preference. We focus here in particular on Keeney's (1976) group cardinal utility axiomatization, which translates to utility theory the conditions put forward by Arrow (1951) for aggregating individual rankings into a social ranking.

The contribution of this work is to revisit and reinterpret the condition of nondictatorship put forward by Arrow (1951) in a way that makes it more consistent with common understanding of what a dictator is. Stronger conditions that limit the maximum influence that any single individual can have are proposed, considering the purpose of the group's decision: either obtaining a full ranking of the alternatives or just selecting a winner. Then, the corresponding new characterizations of group utility functions over certain alternatives and group expected utility functions over uncertain alternatives are derived. In the case of group expected utility 
functions over uncertain alternatives, these characterizations place an upper bound on each individual's weight. An extension of the notion of dictator to coalitions of individuals is also discussed.

\section{The Results of Arrow (1951) and Keeney (1976)}

Arrow (1951) addressed the problem of aggregating $N$ individual rankings into a group ranking. Formally, Arrow considered at the outset binary relations $R_{i}$ such that for any two alternatives $a$ and $b, a R_{i} b$ means that the individual indexed by $i(i=1, \ldots, N)$ either prefers $a$ to $b$ or is indifferent between them. Arrow defined that these binary relations should be weak orders through two axioms stating that $R_{i}$ is connected and transitive. The desideratum for an aggregation method, according to Arrow, would be to obtain a social ranking $R$ that is also connected and transitive. This method should satisfy five seemingly reasonable conditions: universality, positive association of social and individual values, independence of irrelevant alternatives, citizens' sovereignty, and nondictatorship.

Keeney (1976) formulated a group cardinal utility axiomatization for certain and for uncertain alternatives. Formally, Keeney considered at the outset a set of $N$ cardinal utilities $u_{i}\left(a_{j}\right)$ concerning individuals indexed by $i(i=1, \ldots, N)$ and alternatives indexed by $j(j=1, \ldots, M)$, and proposed five assumptions parallel to Arrow's conditions that a group cardinal utility function $u_{G}=u\left(u_{1}, \ldots, u_{N}\right)$ should be consistent with (Keeney 1976, p. 142):

Assumption B1. There are at least two individual members in the group, at least two alternatives, and group utilities are specified for all possible individual member's utilities.

Assumption B2. If the group utilities indicate alternative $a$ is preferred to alternative $b$ for a certain set of individual utilities, then the group utilities must imply a is preferred to $b$ if (i) the individual's utilities of alternatives other than $a$ are not changed and (ii) each individual's utilities for a either remain unchanged or are increased.

Assumption B3. If an alternative is eliminated from consideration, the new group utilities for the remaining alternatives should be equivalent (i.e., positive linear transformations) of the original group utilities for these same alternatives.
Assumption B4. For each pair of alternatives $a$ and $b$, there is some set of individual utilities such that the group prefers $a$ to $b$.

Assumption B5. There is no individual with the property that whenever he prefers alternative $a$ to $b$, the group will also prefer a to $b$ regardless of the other individuals' utilities.

Two main results were proved. In the case of certain alternatives, $u_{G}$ is consistent with those five assumptions if and only if $d u / d u_{i} \geq 0$, for $i=1, \ldots, N$, and the inequality is strict for at least two $u_{i}{ }^{\prime} \mathrm{s}$ (Keeney 1976, Theorem 1). In the case of uncertain alternatives (involving aggregation of von NeumannMorgenstern expected utilities), to be consistent with the five assumptions, $u_{G}$ needs to be a linear combination of individual expected utility functions (Keeney 1976, Theorem 2):

$$
u\left(u_{1}, \ldots, u_{N}\right)=\sum_{i=1}^{N} k_{i} u_{i}
$$

with $k_{i} \geq 0$, for $i=1, \ldots, N$, and the inequality is strict for at least two $k_{i}$ 's. The $k_{i}$ 's are scaling coefficients associated with the individuals.

\section{Strengthening the Nondictatorship Assumption}

Arrow's (1951, p. 30) nondictatorship condition states that "[t]he social welfare function is not to be dictatorial," where dictatorial means that "there exists an individual $i$ such that, for all $a$ and $b, a P_{i} b$ implies $a P b$ regardless of the orderings $R_{1}, \ldots, R_{N}$ of all individuals other than $i$, where $P$ is the social preference relation corresponding to $R_{1}, \ldots, R_{N}$." Let us note that according to this definition, if individual $i$ is a dictator, then $R=R_{i}$.

Keeney (1976) formulated by analogy a nondictatorship condition (Assumption B5 in the previous section). As in Arrow's (1951) case, this assumption implies that if individual $i$ is a dictator, then the group ranking provided by $u_{G}$ coincides with the ranking implicit in $u_{i}$.

The nondictatorship conditions of Arrow and Keeney consider that a dictator is an individual so powerful that for any conceivable pair $(a, b)$ in the space of alternatives (not necessarily the actual alternatives that a group of individuals is considering), 
Dias and Sarabando: A Note on a Group Preference Axiomatization with Cardinal Utility Decision Analysis 9(3), pp. 231-237, ( 2012 INFORMS

Table 1 Hypothetical Utilities of $N$ Individuals

\begin{tabular}{lcccc}
\hline & Individual 1 & Individual 2 & $\cdots$ & Individual N \\
\hline$u_{i}(a)$ & $u_{1}(a)$ & 0 & & 0 \\
$u_{i}(b)$ & $u_{1}(b)$ & 1 & $\ldots$ & 1 \\
$k_{i}$ & $1-\varepsilon$ & $k_{2}$ & & $k_{N}$ \\
\hline
\end{tabular}

if the dictator (an individual $i$ ) deems that $a$ is preferred to $b$, then this yields $a P b$ for the group, no matter how close $u_{i}(a)$ and $u_{i}(b)$ are.

Consider for instance that $u_{G}$ follows the additive model (1), irrespective of addressing certain or uncertain alternatives. Let us also assume that (following a common convention) all utilities are in the $[0,1]$ interval, and the sum of the scaling coefficients is equal to 1:

$$
\forall i, u_{i} \in[0,1], k_{i} \geq 0, \quad \text { and } \quad \sum_{i=1}^{N} k_{i}=1 .
$$

Let us consider an example in which an individual $(i=1)$ has a scaling coefficient arbitrarily close to 1 : $k_{1}=1-\varepsilon$ for a small positive quantity $\varepsilon$. In the situation depicted in Table 1, individual 1 is not a dictator in Keeney's (1976) sense. Indeed, no matter how small $\varepsilon$ is, we can conceive of two alternatives such that $u_{G}(b)>u_{G}(a)$, despite $u_{1}(b)<u_{1}(a)$. For instance, if $u_{1}(a)=c$ and $u_{1}(b)=c-\varepsilon$, then $u_{G}(b)-u_{G}(a)=$ $(1-\varepsilon)(c-\varepsilon)+\varepsilon-(1-\varepsilon) c=\varepsilon^{2}>0$.

Suppose that there are three individuals and $k_{1}=$ $0.9990, k_{2}=0.0005$, and $k_{3}=0.0005$. In this situation, individuals 2 and 3 would arguably consider that individual 1 is a dictator. One might counterargue, claiming that individual 1 is not a dictator because, for instance, if individual 1 had a very slight preference for one alternative $a$ compared to some other alternative $b$, then individuals 2 and 3 might be decisive if they have an extreme preference for $b$ (namely, have utility 1 for $b$ and utility 0 for $a$ ). However, this explanation would hardly convince individuals 2 and 3 that there is no dictatorship.

A reasoning that can lead to the sentiment that these scaling coefficients would make individual 1 a dictator is that it is very easy for individual 1 to impose a winner, or even a whole ranking, regardless of the utilities of all other individuals. For instance, if there are five alternatives $a, b, c, d$, and $e$, and individual 1 declares, for instance, $u_{1}(a)=1, u_{1}(b)=0.75$, $u_{1}(c)=0.5, u_{1}(d)=0.25$, and $u_{1}(e)=0$, then he would impose the ranking $a P b P c P d P e$ even if this totally contradicts the utilities of individuals 2 and 3.

This type of reasoning involves acknowledging the possibility of strategic misrepresentation, but in a way that makes it more difficult to accept socially than what is usually considered in voting theory. In voting theory a method is said to be subject to strategic vote (subject to "manipulation") if an individual might get some benefit by not voting according to his preferences. For instance, an individual can vote for his second choice because he foresees that a worse candidate might win if he votes for his first choice. This is not considered a major drawback because, as Gibbard (1973) and Satterthwaite (1975) have shown, all universal and nondictatorial methods that aggregate individual rankings to produce a social ranking are potentially subject to strategic vote. Furthermore, the fact that an individual might benefit does not guarantee he will benefit: this would require knowing the preferences of the other individuals in advance, and knowing whether these other individuals would also vote strategically.

The type of strategic misrepresentation that we can seek to prevent is arguably much more crucial to the acceptability of a group aggregation model: no individual should be able to indicate his (possibly misrepresented) preferences in a way that it guarantees that his preferences are reproduced by the group utility function regardless of the preferences indicated by all other members. This can be formalized in different ways. We next propose three conditions a group might wish to enforce to avoid such a "strategic dictator."

Condition 1 (ImMunity to Imposition of a RankING BY AN INDIVIDUAL (IIR)). There is no individual with the property that he can indicate preferences (possibly acting strategically) in a way that guarantees that the group's ranking of the alternatives coincides with his complete ranking of the alternatives (without ties), regardless of all other individuals' preferences.

Condition 2 (Immunity to Imposition of a WinNER BY AN INDIVIDUAL (IIW)). There is no individual with the property that he can indicate preferences in a way that guarantees that his preferred alternative has a group utility strictly greater than the utilities of all 
other alternatives, regardless of all other individuals' preferences.

CONDition 3 (IMmUnity to Imposition of a WeAK WINNER bY AN INDIVIDUAL (IIWW)). There is no individual with the property that he can indicate preferences in a way that guarantees his preferred alternative has the highest group utility, possibly indifferent to other alternatives (i.e., other alternatives can be tied for the first place), regardless of all other individuals' preferences.

Note that IIWW implies IIW, which in turn implies IIR, and IIR implies the nondictatorship conditions of Arrow (1951) and Keeney (1976).

\section{Axiomatization}

Although Conditions 1-3 could also be set in Arrow's (1951) context of ordinal aggregation, we focus on the group cardinal utility setting of Keeney (1976).

Let us recall that we are considering 0 , the worst possible utility level, and 1 , the best possible utility level (which is a commonly used scale). Normalizing utility functions (e.g., by means of an affine transformation) so that all utilities are in the interval $[0,1]$ can make these functions dependent on the set of actual alternatives or the set of potential alternatives the group should agree to consider. Dhillon and Mertens (1999) suggest that such normalization should take into account the set of all potential alternatives limited only by feasibility and justice. The propositions that follow do not depend on how the $[0,1]$ normalization is made. Furthermore, because we are admitting the possibility of strategically misrepresenting preferences, assuming a $[0,1]$ interval for utilities merely bounds the utilities that each individual can indicate (rather than have).

Let $u_{G}\left(u_{i}, 0_{-i}\right)$ denote the group utility of an alternative that has utility $u_{i}$ for individual $i$ and utility 0 for all other individuals. Let $u_{G}\left(u_{i}, 1_{-i}\right)$ denote the group utility of an alternative that has utility $u_{i}$ for individual $i$ and utility 1 for all other individuals. The following propositions characterize a group utility function that satisfies Keeney's (1976) assumptions plus immunity to a strategic dictator.

Proposition 1. A group cardinal utility function over certain alternatives with utilities in $[0,1]$ is consistent with Assumptions B1-B4 and IIW if and only if $d u / d u_{i} \geq 0$, for $i=1, \ldots, N$, with strict inequality for at least two $u_{i}$ 's, and there is no individual $i$ such that $u_{G}\left(1_{i}, 0_{-i}\right)>u_{G}\left(0_{i}, 1_{-i}\right)$.

Proof. $(\Leftarrow)$ Assume there is no individual $i$ such that $u_{G}\left(1_{i}, 0_{-i}\right)>u_{G}\left(0_{i}, 1_{-i}\right)$. Then, if $N-1$ individuals assign utility 0 to an alternative $a$ and utility 1 to an alternative $b$, the remaining individuals cannot impose $a$ as a (single) winner. Thus, IIW (which refers to the possibility of imposing any alternative as a single winner) is assured. Keeney's (1976) Theorem 1 proves that $d u / d u_{i} \geq 0$, for $i=1, \ldots, N$, with strict inequality for at least two $u_{i}{ }^{\prime} \mathrm{s}$, is sufficient for Assumptions B1-B4.

$\Rightarrow$ Assume by contradiction that $u_{G}\left(1_{i}, 0_{-i}\right)>$ $u_{G}\left(0_{i}, 1_{-i}\right)$ for some individual $i$. If $i$ 's preferred alternative is $a$ and he states $u_{i}(a)=1$, and for all $b \neq a$ states $u_{i}(b)=0$, then $u_{G}(a)>u_{G}(b)$, imposing $a$ as a winner, even if all other individuals $j \neq i$ state $u_{j}(a)=0$ and for all $b \neq a$ state $u_{j}(b)=1$. Keeney's (1976) Theorem 1 proves the necessity of Assumptions B1-B4.

Proposition 2. A group cardinal utility function over certain alternatives with utilities in $[0,1]$ is consistent with Assumptions B1-B4 and IIWW if and only if $d u / d u_{i} \geq 0$, for $i=1, \ldots, N$, with strict inequality for at least two $u_{i}$ 's and there is no individual $i$ such that $u_{G}\left(1_{i}, 0_{-i}\right) \geq u_{G}\left(0_{i}, 1_{-i}\right)$.

The proof is analogous to that of Proposition 1.

Proposition 3. A group cardinal expected utility function over uncertain alternatives with utilities in $[0,1]$ is consistent with Assumptions B1-B4 and IIW if and only if it has the form (1) and $k_{i} \geq 0$, for $i=1, \ldots, N$, and there is no individual $i$ such that $k_{i}>0.5$.

Proof. This is a corollary of Proposition 1 applying (1) together with Keeney's (1976) Theorem 2. Note that when $k_{i}>0.5$, Equation (1) yields $u_{G}\left(1_{i}, 0_{-i}\right)>$ 0.5 , and, because $k_{1}+\cdots+k_{i-1}+k_{i+1}+\cdots+k_{N}=$ $1-k_{i}$ (recall we assume weights add up to one), Equation (1) yields $u_{G}\left(0_{i}, 1_{-i}\right)<0.5$.

Proposition 4. A group cardinal expected utility function over uncertain alternatives with utilities in $[0,1]$ is consistent with Assumptions B1-B4 and IIWW if and only if it has the form (1) and $k_{i} \geq 0$, for $i=1, \ldots, N$, and there is no individual $i$ such that $k_{i} \geq 0.5$. 
The proof is analogous to that of Proposition 3.

When we consider the IIR condition (immunity to imposition of a ranking by an individual) for group expected utility functions over uncertain alternatives, we can also find a characterization that is easy to verify in practice (Proposition 6 below). However, for the case of utilities over certain alternatives, the characterization we obtain (Proposition 5 below) is less prone to be easily checked. Let us first introduce some additional notation:

Let $M$ denote the number of alternatives to be ranked.

Let $S_{i(1)}=\left\{d_{i} \in[0,1]: u_{G}\left(d_{i}, 0_{-i}\right)>u_{G}\left(0,1_{-i}\right)\right\}$.

If $S_{i(1)} \neq \varnothing$, let $d_{i(1)}=\inf S_{i(1)}$. Then, individual $i$ is able to impose a preference between two of the alternatives $a_{[1]}$ and $a_{[2]}$ by assigning $u_{i}\left(a_{[1]}\right)=d_{i(1)}$ and $u_{i}\left(a_{[2]}\right)=0$, regardless of the other individuals' preferences. Let us now define recursively

$$
S_{i(j+1)}= \begin{cases}\varnothing & \text { if } S_{i(j)}=\varnothing, \\ \left\{d_{i} \in\left[d_{i(j)}, 1\right]:\right. & \\ \left.u_{G}\left(d_{i}, 0_{-i}\right)>u_{G}\left(d_{i(j)}, 1_{-i}\right)\right\} & \text { if } S_{i(j)} \neq \varnothing,\end{cases}
$$

where $d_{i(j)}=\inf S_{i(j)}$.

Thus, if $S_{i(2)} \neq \varnothing$, individual $i$ is able to impose a preference ranking between three of the alternatives $a_{[1]}, a_{[2]}$, and $a_{[3]}$ by assigning $u_{i}\left(a_{[1]}\right)=d_{i(2)}, u_{i}\left(a_{[2]}\right)=$ $d_{i(1)}$, and $u_{i}\left(a_{[3]}\right)=0$, regardless of the other individuals' preferences, and so on. By recursion, if $S_{i(j)} \neq \varnothing$, individual $i$ is able to impose a preference ranking for $j+1$ alternatives.

Proposition 5. A group cardinal utility function over certain alternatives with utilities in $[0,1]$ is consistent with Assumptions B1-B4 and IIR if and only if $d u / d u_{i} \geq 0$, with strict inequality for at least two $u_{i}{ }^{\prime} s$, and $S_{i(M-1)}=\varnothing$, for $i=1, \ldots, N$.

Proof. $(\Leftarrow)$ Assume $S_{i(M-1)}=\varnothing$, for $i=1, \ldots, N$. By construction, this means that no individual can impose a ranking of the $M$ alternatives. Thus IIR (which refers to the possibility of imposing any complete ranking) is assured. Keeney's (1976) Theorem 1 proves that $d u / d u_{i} \geq 0$, for $i=1, \ldots, N$, with strict inequality for at least two $u_{i}$ 's, is sufficient for Assumptions B1-B4.

$(\Rightarrow)$ Assume by contradiction that $S_{i(M-1)} \neq \varnothing$ for some individual $i$. By construction, if $S_{i(M-1)} \neq \varnothing$, individual $i$ is able to impose a ranking $a_{[1]} P a_{[2]}$ $P, \ldots, P a_{[M]}$ by assigning $u_{i}\left(a_{[1]}\right)=d_{i(M-1)}, u_{i}\left(a_{[2]}\right)=$ $d_{i(M-2)}, \ldots, u_{i}\left(a_{[M]}\right)=0$. Keeney's (1976) Theorem 1 proves the necessity of Assumptions B1-B4.

Proposition 6. A group cardinal expected utility function over uncertain alternatives with utilities in $[0,1]$ is consistent with Assumptions B1-B4 and IIR if and only if it has the form (1) and $k_{i} \geq 0$, for $i=1, \ldots, N$, and there is no individual $i$ such that $k_{i}>(M-1) / M$.

Proof. This is a corollary of Proposition 5 applying (1) together with Keeney's (1976) Theorem 2, because $u_{G}\left(\delta+d_{i(j)}, 0_{-i}\right)>u_{G}\left(d_{i(j)}, 1_{-i}\right) \Leftrightarrow \delta k_{i}>\left(k_{1}+\right.$ $\left.\cdots+k_{i-1}+k_{i+1}+\cdots+k_{N}\right)$, which does not depend on $d_{i(j)}$. Therefore, $d_{i(2)}-d_{i(1)}=d_{i(3)}-d_{i(2)}$, and so on. Thus, $S_{i(M-1)} \neq \varnothing$ if and only if $k_{i} /(M-1)-\left(k_{1}+\right.$ $\left.\cdots+k_{i-1}+k_{i+1}+\cdots+k_{N}\right)>0$, allowing individual $i$ to impose a ranking $a_{[1]} P a_{[2]} P, \ldots, P a_{[M]}$ by assigning $u_{i}\left(a_{[1]}\right)=(M-1) /(M-1)=1, u_{i}\left(a_{[2]}\right)=(M-2) /$ $(M-1), \ldots, u_{i}\left(a_{[M-1]}\right)=1 /(M-1)$, and $u_{i}\left(a_{[M]}\right)=0$. Because weights add up to one, $k_{i} /(M-1)-\left(k_{1}+\cdots+\right.$ $\left.k_{i-1}+k_{i+1}+\cdots+k_{N}\right)>0 \Leftrightarrow k_{i} /(M-1)-\left(1-k_{i}\right)>0 \Leftrightarrow$ $k_{i}>(M-1) / M$.

Let us note that IIR refers to the imposition of a complete order. The case of imposing a weak order (where ties are allowed) is not interesting: to impose that a group utility function faithfully reproduces a weak order would imply that $d u / d u_{i}=0$ for all individuals except the dictator, thereby failing to comply with Keeney's (1976) definition of a cardinal group utility function.

\section{Extension to Coalitions of Individuals Acting Strategically}

The notions of imposition of a ranking and imposition of a winner can be extended to a coalition of individuals who strategically act as a group, according to the following definitions:

Imposition of a winner by a coalition. A coalition of individuals $C \subseteq\{1, \ldots, N\}$ who have the same preferred winner (without indifference, i.e., without alternatives tied for the first place) can impose this winner to the rest of the group (without indifference) if the members of $C$ can indicate preferences (possibly acting strategically) in a way that guarantees that the preferred alternative of the members of $C$ has the highest 
group utility, without indifference, regardless of all other individuals' preferences.

Imposition of a weak winner by a coalition. This is the same as the previous definition, but allowing indifference, i.e., allowing other alternatives to be tied for the first place.

Imposition of a ranking (complete order) by a coalition. A coalition of individuals $C \subseteq\{1, \ldots, N\}$ who have the same preferred ranking can impose this ranking to the rest of the group if the members of $C$ can indicate preferences in a way that guarantees the (overall) group's ranking of the alternatives coincides with the preferred ranking of the members of $C$, regardless of all other individuals' preferences.

For brevity we will focus on the notion of imposition of a winner and on the case of a group cardinal expected utility function over uncertain alternatives. The following results characterize expected utility functions seeking to avoid a dictatorial coalition.

Proposition 7. Given the conditions of Proposition 3, a coalition of individuals $C \subseteq\{1, \ldots, N\}$ who have the same preferred winner can impose this winner to the rest of the group (let $\bar{C}=\{1, \ldots, N\}-C$ ) if and only if $\sum_{i \in C} k_{i}>0.5$.

Proof. $(\Leftarrow)$ If $\sum_{i \in C} k_{i}>0.5$ and individuals in $C$ assign utility 1 to an alternative $a$, then $u_{G}(a)=$ $\sum_{i \in C} k_{i}+\sum_{i \in \bar{C}} k_{i} u_{i}(a)>0.5$. If the same individuals assign utility 0 to an alternative $b$, then $u_{G}(b)=$ $\sum_{i \in \bar{C}} k_{i} u_{i}(b) \leq \sum_{i \in \bar{C}} k_{i}<0.5$ (because $\sum_{i \in \bar{C}} k_{i}=1-$ $\left.\sum_{i \in C} k_{i}\right)$. Hence, $u_{G}(a)>u_{G}(b)$; i.e., individuals in $C$ are able to impose $a$ as a winner.

$(\Rightarrow)$ Assume by contradiction that $\sum_{i \in C} k_{i} \leq 0.5$. If individuals not in $C$ assign utility 0 to an alternative $a$, then $u_{G}(a) \leq \sum_{i \in C} k_{i} \leq 0.5$. If the same individuals assign utility 1 to an alternative $b$, then $u_{G}(b) \geq$ $\sum_{i \in \bar{C}} k_{i} \geq 1-0.5$. In such a case, $u_{G}(a) \leq u_{G}(b)$; i.e., individuals in $C$ are not able to impose $a$ as a (single) winner.

Corollary of Proposition 7. Given the conditions of Proposition 3, there is no coalition of $N_{c}$ individuals capable of imposing their preferred winner to the group if $k_{i} \leq 0.5 / N_{c}$ for $i=1, \ldots, N$.

Proof. If $k_{i} \leq 0.5 / N_{c}$ for all individuals and there are $N_{c}$ individuals in $C$, then $\sum_{i \in C} k_{i} \leq 0.5$, and hence, by Proposition 6, coalition $C$ cannot impose a (single) winner.

Proposition 8. Given the conditions of Proposition 3, a coalition of individuals $C \subseteq\{1, \ldots, N\}$ who have a preferred winner (possibly indifferent to other alternatives) in common can impose this weak winner to the rest of the group if and only if $\sum_{i \in C} k_{i} \geq 0.5$.

The proof is analogous to that of Proposition 7.

Corollary of Proposition 8. Given the conditions of Proposition 3, there is no coalition of $N_{c}$ individuals capable of imposing their preferred winner (possibly indifferent to other alternatives) to the group if $k_{i}<0.5 / N_{c}$ for $i=1, \ldots, N$.

The proof is analogous to that of the corollary of Proposition 7.

Proposition 9. Given the conditions of Proposition 3, if there is no coalition of $N_{c}$ individuals capable of imposing their preferred (single) winner to the group, then any coalition of $N-N_{c}$ individuals can impose a weak winner.

Proof. Because $\sum_{i \in C} k_{i}+\sum_{i \in \bar{C}} k_{i}=1$, if $\sum_{i \in C} k_{i} \leq 0.5$, then $\sum_{i \in \bar{C}} k_{i} \geq 0.5$, and the reasoning of Propositions 7 and 8 applies.

This last result indicates that there will always be at least one subset of individuals of a group that might act strategically to impose a winner to the whole group. In particular, if we guarantee that an individual cannot act strategically to impose a winner, then the remaining $N-1$ individuals might act strategically impose a winner to this individual; if two individuals cannot impose a winner, then a group of $N-2$ individuals might do so, etc. If $\lfloor N / 2\rfloor$ (the integer part of $N / 2$ ) individuals cannot impose a winner, then a group of $\lfloor N / 2\rfloor+1$ individuals might do so. In other words, a simple majority of the individuals will always be able to impose a winner if this majority acts strategically.

If it is requested that a coalition that is not a simple majority can never act strategically to impose a (single) winner, then it is necessary to have $k_{i} \leq$ $0.5 /(\lfloor N / 2\rfloor)(i=1, \ldots, N)$. This means a limit $k_{i} \leq 0.5$ if $N=2$ or $N=3, k_{i} \leq 1 / 4$ if $N=4$ or $N=5$, etc. As $N$ increases, this limit tends to $1 / N$. 


\section{Concluding Notes}

We introduced a concept of strategic dictator that is consistent with the common understanding of that term and derived new conditions for a group utility function. Three types of dictatorial situations were addressed: the possibility of imposing a complete ranking of the alternatives, the possibility of imposing a single winner to the group, and the possibility of imposing a weak winner (possibly tied with other alternatives having the same maximal utility). Avoiding these possibilities entails adding successively more stringent conditions to Keeney's (1976) conditions for a group utility function. Keeney's conditions, on the other hand, already prevented the possibility of imposing a weak ranking of the alternatives by a dictator due to the monotonicity assumption B2.

The analysis was extended to consider the imposition of a winner by a coalition of individuals acting strategically. We have shown that this is impossible to avoid: if an individual is not a dictator, then the coalition of the remaining individuals is dictatorial. However, most people would not consider this to be a problem. Moreover, in practice it is more difficult for individuals to act strategically together (in collusion) than it is for a single individual to act strategically on his own. Therefore, it may not be warranted to constrain the group utility function to prevent the imposition of a winner by a coalition of more than half of the individuals. Nevertheless, in some situations, preventing a dictatorial coalition of fewer individuals (e.g., $1 / 3$ of the group), or at least preventing a strategic dictator (by imposing IIW) will be considered a must-have feature for a group utility function.

\section{Acknowledgments}

The authors thank Ralph Keeney for commenting on an earlier draft of this paper and offering suggestions that motivated §5. The helpful remarks of two anonymous referees are also gratefully acknowledged. This work was partially supported by the COMPETE program and the
Portuguese Foundation for Science and Technology [Projects FCOMP-01-0124-FEDER-MIT/MCA/0066/2009, MIT/SET/ 0014/2009, PEst-C/EEI/UI0308/2011].

\section{References}

Arrow K (1951) Social Choice and Individual Values (John Wiley \& Sons, New York).

Baucells M, Sarin R (2003) Group decisions with multiple criteria. Management Sci. 49(8):1105-1118.

Bose U, Davey AM, Olson DL (1997) Multi-attribute utility methods in group decision making: Past applications and potential for inclusion in GDSS. Omega 25(6):691-706.

Dhillon A, Mertens J-F (1999) Relative utilitarianism. Econometrica 67(3):471-498.

Dyer J, Sarin R (1979) Group preference aggregation rules based on strength of preference. Management Sci. 25(9):822-832.

Gibbard A (1973) Manipulation of voting schemes: A general result. Econometrica 41(4):587-601.

Harsanyi J (1955) Cardinal welfare, individualistic ethics, and interpersonal comparisons of utility. J. Political Econom. 63(4):309-321.

Harvey C (1999) Aggregation of individuals' preference intensities into social preference intensity. Soc. Choice Welfare 16(1):65-79.

Keefer DL, Kirkwood CW, Corner JL (2004) Perspective on decision analysis applications, 1990-2001. Decision Anal. 1(1):4-22.

Keeney RL (1976) A group preference axiomatization with cardinal utility. Management Sci. 23(2):140-145.

Keeney RL, Kirkwood CW (1975) Group decision making using cardinal social welfare functions. Management Sci. 22(4): 430-437.

Kilgour DM, Eden C, eds. (2010) Handbook of Group Decision and Negotiation (Springer, New York).

Merrick JRW, Parnell GS, Barnett J, Garcia M (2005) A multipleobjective decision analysis of stakeholder values to identify watershed improvement needs. Decision Anal. 2(1):44-57.

Phillips LD, Bana e Costa C (2007) Transparent prioritisation, budgeting, and resource allocation with multi-criteria decision analysis and decision conferencing. Ann. Oper. Res. 154(1):51-68.

Satterthwaite M (1975) Strategy-proofness and Arrow's conditions: Existence and correspondence theorems for voting procedures and social welfare functions. J. Political Econom. 10(2):187-217.

Schein ES (1999) Process Consultation Revisited (Addison-Wesley, Reading, MA).

von Neumann J, Morgenstern O (1947) Theory of Games and Economic Behaviour, 2nd ed. (Princeton University Press, Princeton, NJ). 